\title{
Predictors of the voltage derived left atrial fibrosis in patients with long-standing persistent atrial fibrillation
}

\author{
Radoslaw M. Kiedrowicz, Maciej Wielusinski, Andrzej Wojtarowicz, Jaroslaw Kazmierczak \\ Cardiology Department, Pomeranian Medical University, Szczecin, Poland
}

\begin{abstract}
Background: Left atrial (LA) arrhythmogenic substrate beyond the pulmonary veins (PV) seems to play a crucial role in the maintenance of atrial fibrillation $(A F)$. The aim of this study was to evaluate the association of selected parameters with the presence and extent of voltage-defined LA fibrosis in patients with long-standing persistent $A F$ (LSPAF) undergoing catheter ablation.

Methods: One hundred and sixteen consecutive patients underwent high density-high resolution voltage mapping of the LA with a multielectrode catheter following PV isolation and restoration of sinus rhythm with cardioversion. A non-invasive dataset, such as clinical variables, two- and three-dimensional echocardiography determined LA size and function and fibrillatory-wave amplitude on a standard surface electrocardiogram were obtained during $A F$ before ablation.

Results: Low-voltage areas (LVA; $15 \mathrm{~cm}^{2}$ [IQR 8-31]) were detected in $56 \%$ of patients. Twenty nine percent of them presented mild, $43 \%$ moderate and $28 \%$ severe global LVA burden. In univariate analysis, age $\geq 57$ years old, female sex, body surface area $\leq 1.76 \mathrm{~m}^{2}$, valvular heart disease, moderate mitral regurgitation, chronic coronary syndrome, hypothyroidism, $\mathrm{CHA}_{2} D \mathrm{~S}_{2}$-VASc score $\geq 3$ and $\geq 4$ predicted the presence of LVA. In multivariate analysis only female sex, valvular heart disease and $\mathrm{CHA}_{2} D \mathrm{~S}_{2-}$ $V A S c \geq 4$ remained statistically significant. $A F$ duration, $L A$ size and function and fibrillatory-waves amplitude were neither associated with the prediction of the LVA, nor severe LVA burden.

Conclusions: A LSPAF diagnosis does not indicate the presence of voltage defined fibrosis in many cases. Simple non-invasive screening of the LSPAF population could predict LVA prevalence. (Cardiol J 2022; 29, 4: 660-669)
\end{abstract}

Key words: atrial fibrillation, long-standing persistent atrial fibrillation, voltage mapping, left atrial fibrosis, low-voltage areas

\section{Introduction}

Left atrial (LA) arrhythmogenic substrate beyond the pulmonary veins (PVs) seems to play a crucial role in the maintenance of atrial fibrillation (AF). Bipolar voltage mapping has been shown to be a useful method to assess the incidence of low-voltage areas (LVA), most commonly considered a marker for the presence of atrial fibrosis [1]. However, the incidence of voltage-derived LA remodelling in patients with long-standing persistent AF (LSPAF), as well as factors that may noninvasively unmask
LVA, has not been thoroughly investigated. The aim of this study was to evaluate the presence and extent of voltage defined LA fibrosis among an LSPAF population by creating high-density high-resolution contact voltage maps acquired with a multielectrode catheter. Moreover, to correlate LVA burden with clinical variables, two- (2D) and three-dimensional (3D) echocardiography determined LA size and function and fibrillatory waves (f-waves) amplitude on a standard surface electrocardiogram (ECG) in order to check the feasibility of noninvasively predicting the presence of an arrhythmogenic substrate.

Address for correspondence: Radoslaw M. Kiedrowicz, MD, PhD, Cardiology Department, Pomeranian Medical University, ul. Powstanców Wlkp. 72, 70-111 Szczecin, Poland, tel: +48 509395560; fax: +48 914661379, e-mail: radkied@wp.pl

Received: 14.11.2019 Accepted: 21.04.2020 Early publication date: 15.05.2020

This article is available in open access under Creative Common Attribution-Non-Commercial-No Derivatives 4.0 International (CC BY-NC-ND 4.0) license, allowing to download articles and share them with others as long as they credit the authors and the publisher, but without permission to change them in any way or use them commercially. 


\section{Methods}

\section{Study population}

The prospective cohort study included 116 consecutive patients with continuous AF of duration greater than 12 months who had undergoing radiofrequency ablation at the documented center. Patients with any previous ablation for AF, cardiac surgery affecting the atria, severe valvular disease or mechanical valve, known pulmonary hypertension, history of myocarditis or pericarditis were excluded. The clinical characteristics of the overall population is summarized in Table 1 . The study complied with the Declaration of Helsinki, all patients provided written, informed consent and the study protocol was approved by a local institutional review board.

\section{Echocardiography examination}

Transthoracic echocardiography was performed on the day of the ablation using a Vivid E9 ultrasound system (GE Vingmed Ultrasound AS) by a single experienced echocardiographist. All 2D and M-mode measurements of the left atrium and ventricle were performed according to recent recommendations [2]. Valvular heart disease was considered as the presence of any moderate regurgitation exclusively. 3D LA volume analysis was made using the dedicated image processing software 4D auto LVQ (GE Healthcare), adjusted manually and corrected using a volume waveform tool. 3D LA systolic (emptying fraction, stroke volume) and diastolic (expansion index) function were calculated by system software. All echocardiographic variables were indexed to body surface area (BSA) where appropriate.

\section{Atrial f-waves amplitude measurement}

A standard surface ECG at the lead gain of $1 \mathrm{mV} / 10 \mathrm{~mm}$ and sweep speed of $50 \mathrm{~mm} / \mathrm{s}$ was analysed. F-wave amplitude was measured on V1 precordial lead with computer-assisted electronic calliper software (Cardio Calipers, Iconico) from wave peak-to-trough by a single physician. The maximal, minimal and mean amplitude, as well as amplitude dispersion of all measured $\mathrm{f}$-waves in a single 5-second ECG recording was reported, except $\mathrm{f}$-waves which overlapped with $\mathrm{QRS}$ and $\mathrm{T}$ waves and was indexed to BSA. Mean f-waves amplitude $<0.1 \mathrm{mV}$ was considered as fine when $\geq 0.1 \mathrm{mV}$ was a coarse AF pattern [3].

\section{Voltage mapping protocol}

An LA respiration-gated shell was created using CARTO $^{\circledR} 3$ electroanatomical platform (Bio-
sense-Webster) with the geometry filling threshold set at 16 using a Pentaray duodecapolar catheter with a 2-6-2 mm electrode spacing configuration (Biosence-Webster) which offers the highest mapping resolution among all multipolar catheters that work with the CARTO3 system. The mitral annulus was defined with a ThermocoolSmartTouch catheter (Biosence-Webster) by electrogram characteristics (local atrial-ventricular amplitude ratio $<0.1$ with a ventricular electrogram $>1.5 \mathrm{mV}$ ). The ventricular portion of the shell was always erased to avoid an overestimation of the total LA surface area (TSA). An encircling isolation of ipsilateral PV pairs (PVI), uniformly delivered $\leq 15 \mathrm{~mm}$ away from the PVs ostia, was performed as the initial step in all patients with a SmartTouch catheter. Then, a direct current shock was applied to restore sinus rhythm in all patients. If $\mathrm{AF}$ failed to be cardioverted or recurred shortly following cardioversion ( $\mathrm{n}=12.9 \%$ of the total study population), the subject was excluded from analysis. Finally, 116 patients were found to be suitable for further evaluation. Following confirmation of PVI in sinus rhythm, a high-density (2876 \pm \pm 1058 points per map), high-resolution bipolar LA voltage mapping, during proximal coronary sinus pacing at $600 \mathrm{~ms}$ cycle length, with a Pentaray catheter acquired with a CONFIDENSE ${ }^{\mathrm{TM}}$ module (Biosence-Webster) was performed. To ensure detailed mapping the distance filling threshold was set at $5 \mathrm{~mm}$, the density acquisition filter at $1 \mathrm{~mm}$ and catheter location stability at $4 \mathrm{~mm}$. A tissue proximity filter was always enabled during mapping in order to reject points not found to be in close proximity to the tissue. Point collection was only allowed when both bipoles on a single spline had adequate catheter-tissue contact. Moreover, internal point filter software was used to limit data acquisition. Only mapping sites that were within a distance of $5 \mathrm{~mm}$ from the acquired LA shell contributed to the voltage map. Further discrete voltage mapping using a SmartTouch catheter, covering less than $10 \%$ of the TSA, at sites presenting inadequate Pentaray-tissue contact was performed if necessary. Electrograms were only accepted if contact force was $\geq 6 \mathrm{~g}$ and catheter location stability did not exceed $2 \mathrm{~mm}$. Electrogram amplitude $\geq 0.5 \mathrm{mV}$ was defined as normal and $<0.5 \mathrm{mV}$ as both moderately and severely diseased tissue [4]. All points presenting low voltage were visually inspected and those incorrectly annotated were deleted from the map in the presence of atrial ectopy, uncaptured coronary sinus pacing, noise, ventricular and atrial farfield. All gaps in the map 


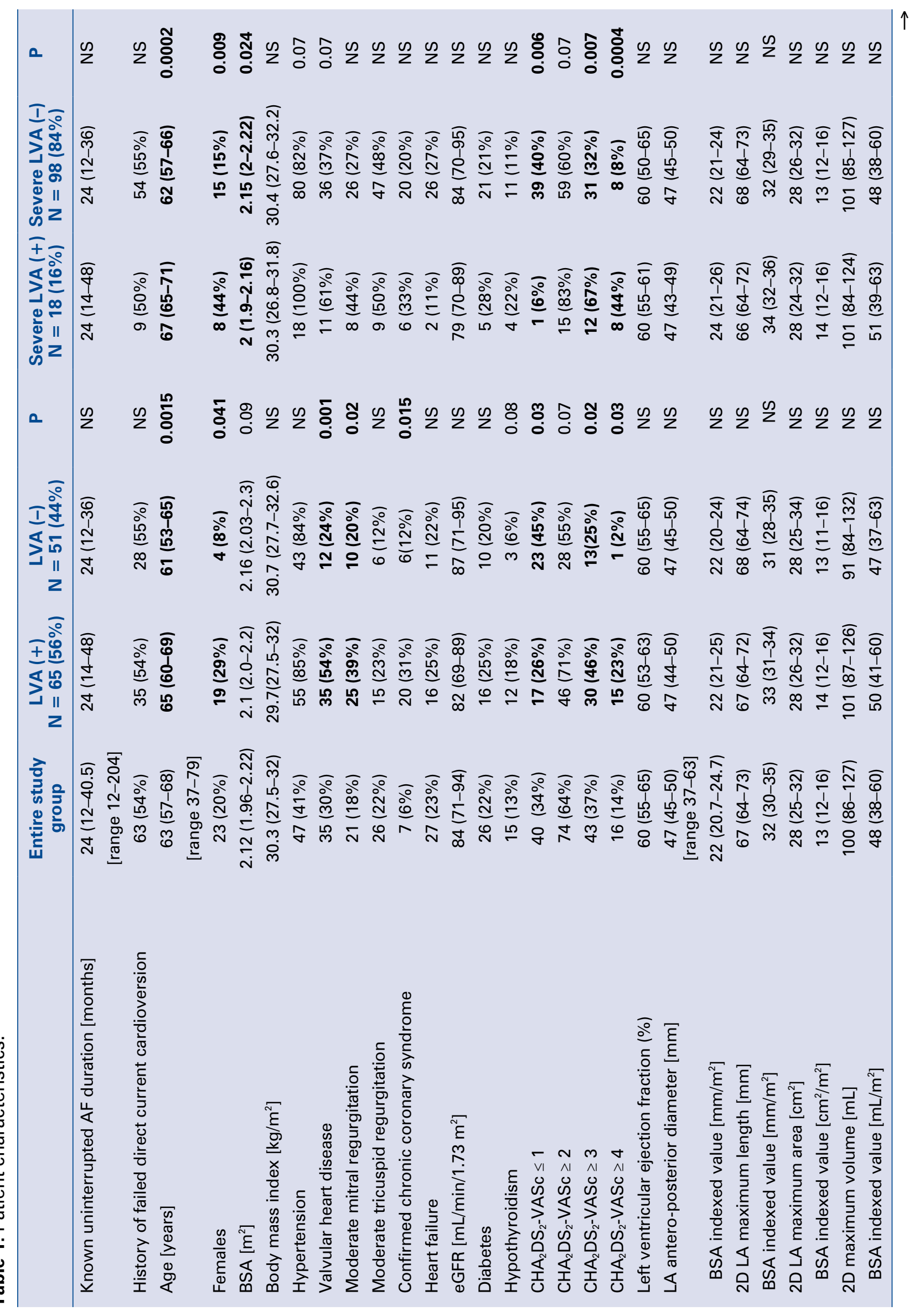




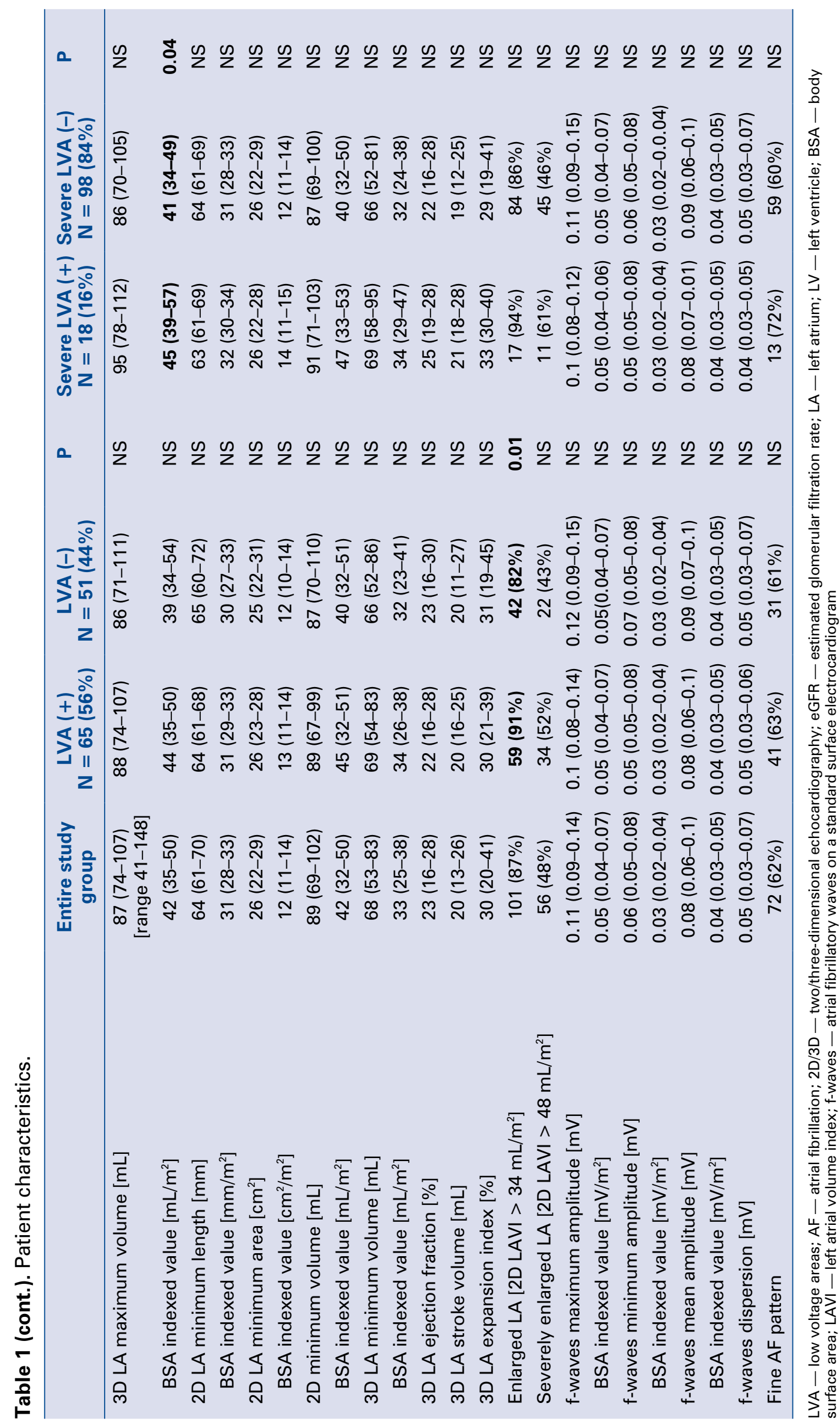




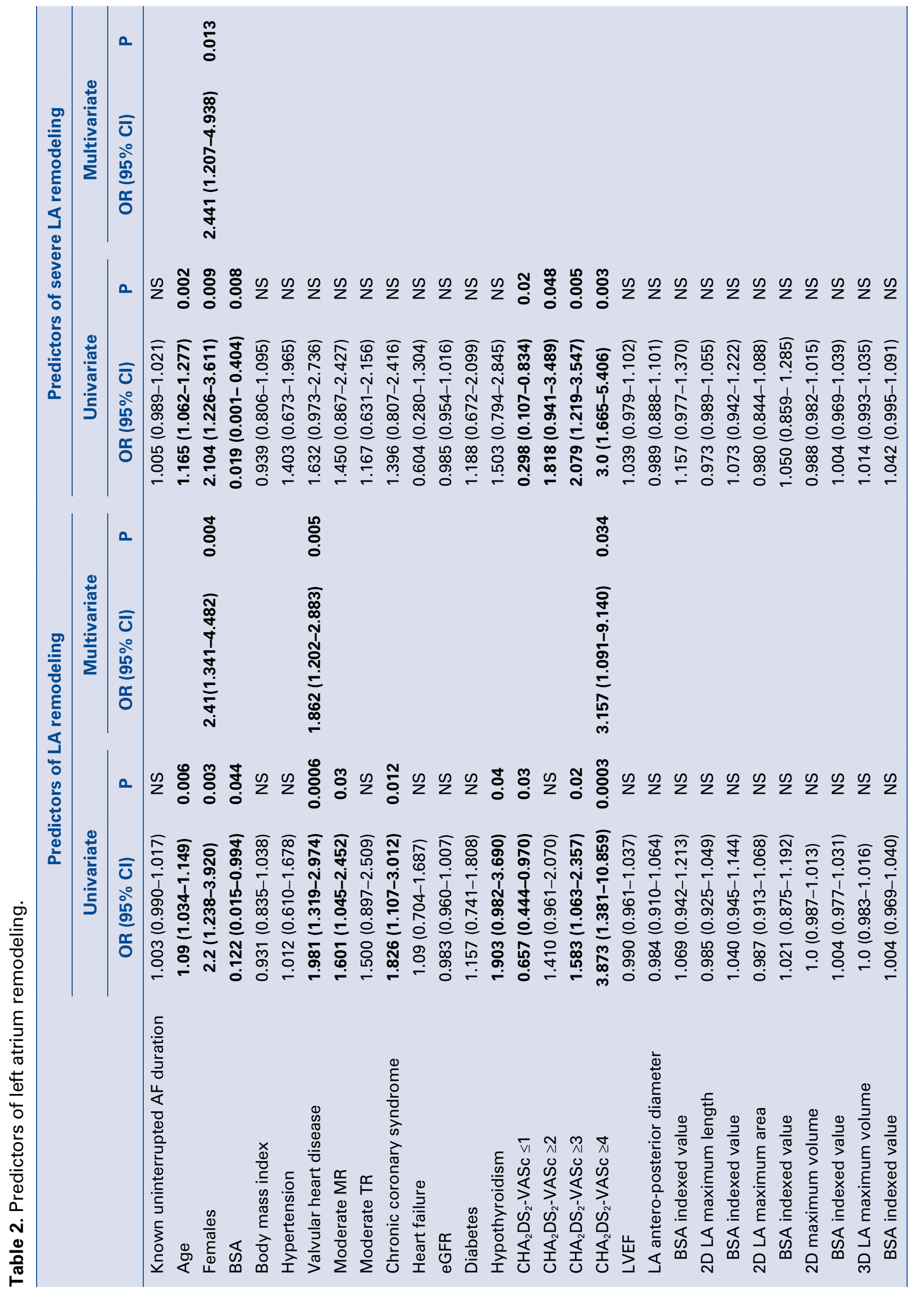




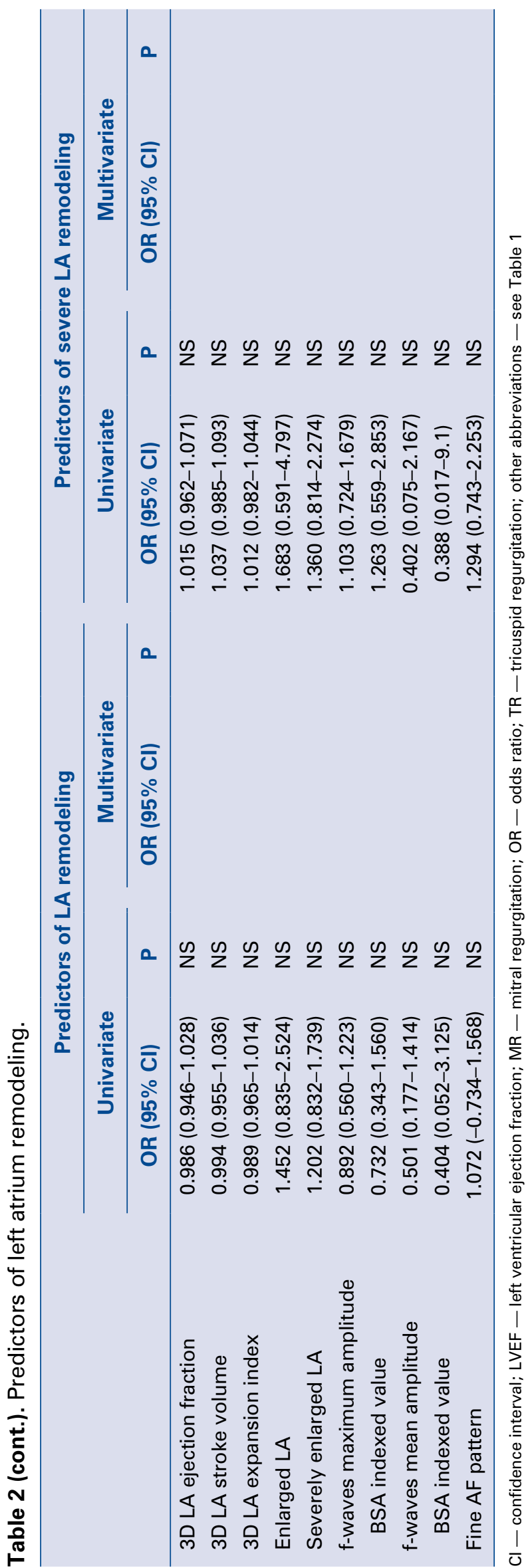

were filled and areas of apparent low voltage were confirmed using an ablation catheter. Extension of all areas showing low-voltage potentials at least $5 \mathrm{~mm}$ away from the ablation lesion set was measured with dedicated CARTO3 system software. The global LVA burden was calculated as the sum of all LVA and then expressed as the percentage of TSA. It was decided to exclude the following areas from TSA calculations: (a) tubular and antral portion of PVs inside the ablation encirclement, (b) a left atrial appendage (LAA), which, in the majority of cases, contributes a great deal to TSA and has been always found to present high voltage in the present study cohort, (c) an area adjacent to the fossa ovalis that always presents low voltage as containing little myocardium. The appendage was defined as an anatomical structure around the LAA orifice, determined internally from within the LA in a reconstructed shell.

The extent of global LVA burden was arbitrarily considered as mild ( $<5 \%$ of the TSA), moderate (5-20\% of the TSA) and severe ( $>20 \%$ of the TSA) on the basis of current observation that all detected LVA can be easily ablated if occupying less than $20 \%$ of the TSA.

The LA was segmented into five areas, i.e. septum, anterior, posterior, inferior and lateral wall and LAA adopting the landmarks proposed by Huo et al. [5].

\section{Statistical analysis}

Continuous data with non-normal distribution is expressed as median and interquartile range (IQR). The categorical variables are presented as values and percentages. Comparisons between groups were performed with either the Mann-Whitney U-test or the $\chi^{2}$ test as appropriate. Univariate and multivariate logistic regression analyses were used to determine factors associated with the existence of LVA. Only variables with a p-value of $<0.05$ in univariate analysis were included for further evaluation in a multivariate model, using a stepwise forward regression. Receiver operating characteristic analysis was used to determine the optimal cut-off value to predict the existence of LVA. Statistical significance for all tests was accepted at a $\mathrm{p}$ value $<0.05$. A statistical analysis was performed using the Statistica 13.3 software (StatSoft).

\section{Results}

Low-voltage areas $\left(15 \mathrm{~cm}^{2}\right.$ [8-31]; $11 \%$ [5-22] of the TSA) were detected in $56 \%$ of the patients. Twenty nine percent of patients with LVA presented mild, $43 \%$ moderate and $28 \%$ severe global LVA 
burden. Fifty-seven percent of patients with LVA presented a disseminated pattern of remodeling including at least $3 \mathrm{LA}$ segments. In $3 \%$ of patients LVA were limited to 2 segments, and a single segment was affected in $29 \%$ (90\% the posterior wall, $5 \%$ the anterior wall and 5\% the inferior wall). The posterior wall was involved in $78.5 \%$ of cases $\left(6 \mathrm{~cm}^{2}\right.$ [5-13]), the anterior LA in $52.3 \%\left(8 \mathrm{~cm}^{2}\right.$ [3-12]), the septum in $49.2 \%\left(8 \mathrm{~cm}^{2}\right.$ [3-11]), the inferior wall in $40 \%\left(8 \mathrm{~cm}^{2}[4-10]\right)$, and the lateral LA in $23 \%$ [4.3 $\mathrm{cm}^{2}$ [3-8]). The lateral LVA was only noted when there was already LVA elsewhere.

Patients with LVA were more frequently female, older, presented valvular heart disease, moderate mitral regurgitation, chronic coronary syndrome, $\mathrm{CHA}_{2} \mathrm{DS}_{2}$-VASc score $\geq 3$ and $\geq 4$ and enlarged LA whilst less often $\mathrm{CHA}_{2} \mathrm{DS}_{2}$-VASc score $\leq 1$ (Table 1 ).

In the univariate analysis, more advanced age, female sex, lower BSA values, valvular heart disease, moderate mitral regurgitation, chronic coronary syndrome, hypothyroidism, $\mathrm{CHA}_{2} \mathrm{DS}_{2}$ VASc score $\geq 3$ and $\geq 4$ predicted the presence of LVA. However, $\mathrm{CHA}_{2} \mathrm{DS}_{2}$-VASc score $\leq 1$ predicted the absence of LVA. A cut-off value of 57 years old predicted LVA incidence with $90 \%$ sensitivity and $65 \%$ specificity. Whereas, $1.76 \mathrm{~m}^{2} \mathrm{BSA}$ cut-off value with $100 \%$ sensitivity and specificity. In the multivariate analysis, only female sex, valvular heart disease and $\mathrm{CHA}_{2} \mathrm{DS}_{2}$-VASc score $\geq 4$ remained statistically significant (Table 2 ).

Patients with severe LVA were more often female, older, presented lower BSA values, $\mathrm{CHA}_{2} \mathrm{DS}_{2}$-VASc score $\geq 3$ and $\geq 4$, higher 3D LA indexed maximum volume, whilst less frequently $\mathrm{CHA}_{2} \mathrm{DS}_{2}$-VASc score $\leq 1$ (Table 1 ).

The severe LVA burden was associated with older age, female sex, lower BSA values, $\mathrm{CHA}_{2} \mathrm{DS}_{2}$ -VASc score $\geq 2, \geq 3$ and $\geq 4$ in the univariate analysis. $\mathrm{CHA}_{2} \mathrm{DS}_{2}$-VASc score $\leq 1$ predicted the absence of severe LVA. A cut-off value of 64 years old predicted severe LVA incidence with $89 \%$ sensitivity and $39 \%$ specificity, whereas $1.89 \mathrm{~m}^{2}$ BSA cut-off value with $89 \%$ sensitivity and $88 \%$ specificity. In the multivariate analysis, only female sex remained statistically significant (Table 2 ).

Atrial fibrillation duration, LA and LV size and function, f-wave amplitude, AF ECG patterns were neither associated with prediction of the LVA nor severe LVA burden.

\section{Discussion}

The key findings of the study were that LSPAF diagnosis does not necessarily equate to extensive voltage-derived LA remodeling and that the best predictors of LVA were female sex, $\mathrm{CHA}_{2} \mathrm{DS}_{2}$-VASc score $>4$ and valvular heart disease. According to available research, this is the first attempt to assess the incidence of voltage-derived LA fibrosis among a large unselected LSPAF population undergoing $\mathrm{AF}$ ablation and to correlate LVA burden with non-invasive pre-ablation parameters. Contemporary data concerning the incidence of voltage-defined LA remodeling describe paroxysmal [6-9], persistent [10-16] or a mixed AF population $[4,5,17-27]$ in which LSPAF patients are regularly underrepresented. Moreover, patients with severely enlarged atria, very long AF duration, who are elderly, with moderate valvular regurgitation or heart failure are commonly excluded $[1,6,8,10,11,13,14,19$, 27]. In addition, presented herein, is a standardized mapping protocol in order to optimize data accuracy.

\section{The prevalence and distribution of LVA}

In previous studies the prevalence of LVA was $10-63 \%$ in paroxysmal AF (PAF) $[4,8,18$, $19,20,25]$ and $35-100 \%$ in persistent AF (PsAF) population $[4,10,12,17,18,20,25]$. The mean extent of LVA was $5-45 \mathrm{~cm}^{2}$ in PAF $[1,18,20$, 25] and $12-72 \mathrm{~cm}^{2}$ in PsAF $[1,12,13,15,16,18$, $20,25]$ when reported. In the present study LVA burden was relatively low and there are at least two possible explanations for this. 1) It might be attributed to the voltage mapping approach. The lack of standardized methodology for defining LVA results in significant heterogeneity in voltage mapping strategies among studies, in particular rhythm during mapping, pre or post PVI analysis, mapping density and resolution, catheter-tissue contact verification, analysis of automatically acquired points, and finally electrogram amplitude cut-off value. It is well known that multielectrode mapping catheters with a small electrodes size and spacing provide much higher mapping resolution of an atrial scar [24]. The accuracy of voltage mapping could further increase with catheter-tissue contact verification and manual point verification [25] and finally high-density acquisition [24]. All of the issues were incorporated into the current approach. 2) This might reflect a heterogeneity of the atrial substrate among the AF population.

In the present cohort, LVA were most often located at the posterior wall which is not in line with other studies, where the anterior wall and septum were generally affected $[1,4,7,8,11,13,15-17,20$, $23,24]$. Moreover, the posterior wall was the most common single remodeling site. Therefore, it can 
be speculated that voltage-derived fibrosis begins at the posterior wall and spreads gradually around the LA. Furthermore, lateral LA, usually a very rare location of LVA $[4,8,11,20,23]$ was affected in relatively many cases, but was never found at a single remodeling site. It can be hypothesized that it is the last affected area when the disseminated pattern of LVA is present. LVA inside the LAA was not found as this was previously reported $[11,20]$.

\section{The predictors of LVA}

Previous studies have shown evidence of LVA with several markers $[1,4,7,8,12,15,18,20$, 22-26]. Intuitively voltage-defined LA remodeling burden would be expected to increase with a longer AF duration time, increased atrial size, decreased LA function, advanced age, the presence of structural heart disease or many concomitant comorbidities and a fine AF pattern. Further, aforementioned discussion about these potential correlations in the light of the present study results was undertaken.

Many studies demonstrated that there is a positive association between LVA and AF persistence $[1,4,7,13,18,20,22,24]$. The underlying mechanism decreasing LA voltage is usually explained by tachycardia induced functional changes that over time result in electrical and structural atrial remodeling [28]. However other studies demonstrated LVA among the PAF population $[1,4,6-9,17-23]$. It was also reported that even the successful elimination of AF fails to halt the progression of fibrosis [29], suggesting that abnormal LA substrate is not the result of arrhythmia alone. Additionally, some studies indicated that the persistence of AF was not a marker of LVA $[15,17,23$, $25,26]$. These findings are in line with the present study results, as there was no correlation between AF duration and detection of LVA. This indicates that there are other factors causing atrial remodeling beside AF and atrial structural changes that could be the cause, and not the consequence of AF.

Many studies reported an association between LA enlargement and LVA [3, 8, 13, 15, 23-27] expressed with LA diameter, area or volume. In the current dataset there was no association between LA size expressed in many various parameters and LVA, despite the fact that $87 \%$ of patients had enlarged and $48 \%$ severely enlarged LA based on widely accepted 2D indexed LA volume [2]. A detailed LA size assessment with 3D echocardiography, which is more accurate than $2 \mathrm{D}$ echocardiography and correlates well with cardiac computed tomography and magnetic resonance imaging [2], did not affect the results. Moreover,
3D derived LA systolic and diastolic function was not associated with LVA. However, due to the limited normative data describing LA function [2] it is hard to assess if the patients presented a decreased LA function pattern. A possible explanation of the present findings is that LVA might be attributed to LA structural, rather than functional remodeling. It was observed that LA remodeling in the AF population, manifesting as a change in atrial size, differs from the consequence of other causes, as it is at least partially reversible [27]. In the current study cohort lack of LVA despite LA enlargement was limited to patients without underlying structural heart disease and it can be speculated that LA enlargement resulted exclusively from LSPAF. Alternatively, the presence of structural heart disease, such as any moderate valvular regurgitation (primary or functional due to annular dilatation as the consequence of LA enlargement [30]) probably resulted in voltage-defined LA fibrosis. LA enlargement was secondary to this scenario as a consequence of valvular regurgitation, $\mathrm{AF}$ or a combination of both. A direct pathophysiological relationship of mitral regurgitation with LA LVA seems obvious, however such a relationship with tricuspid regurgitation is not easy to explain. This could be a manifestation of long-standing increased pulmonary pressure and LA pressure overload [31].

There are some studies that found an association between LVA and age $[1,4,7,13,15,23-26]$. However, the data seem to be ambiguous [8,20]. The present study showed that age does not correlate with LVA incidence which supports the hypothesis that any age contribution to voltagederived remodeling development is limited.

In the majority of studies there is an association between LVA and female sex $[1,4,7,8,15,20$, 24]. The findings herein, are in line with this data. It could be assumed that females are genetically favored for atrial fibrosis [32] and/or undergo AF ablation in an advanced state of the disease [33].

In the present dataset many classical risk factors failed to predict LVA in multivariate analysis. However, a combination of these factors expressed as a high $\mathrm{CHA}_{2} \mathrm{DS}_{2}$-VASc score $>4$ was in fact predictive. This highlights the multifactorial nature of LVA development and the interplay between risk factors. In previous studies a mean risk score of 2.5-2.6 was an independent predictor for LVA $[7,13,18]$ or remained not predictive at all [34].

Fibrillatory wave amplitude on surface ECG could potentially unmask atrial LVA as it is dependent on the magnitude of the underlying voltage, which is related to the magnitude of remaining viable 
atrial muscle [3]. However, in this study such a correlation was not found. What may be considered one major factor, is that atrial activity recorded in lead V1 does not reflect left atrial activity exclusively, but rather right atrial or global atrial activity.

\section{Limitations of the study}

Voltage mapping was limited to patients who were able to maintain sinus rhythm following PVI and cardioversion. Therefore, this may have reduced the overall LVA burden. Voltage mapping following PVI may have excluded a part of LA with low voltages and could have reduced the overall LVA burden. Voltages $<0.5 \mathrm{mV}$ were considered to correlate well with different degrees of LA structural defect, based on previous descriptions. However, this cut-off value has not been clearly validated. It is too early to exclude the extent to which the LA fibrosis in our patient cohort might have been detected or reclassified to normal when compared to other methods for detecting LA fibrosis, especially cardiac magnetic resonance imaging [35]. Females were strongly underrepresented in our population.

\section{Conclusions}

The present study showed that a diagnosis of LSPAF does not indicate the presence of LVA in many cases and that neither long AF duration, LA enlargement, nor ECG parameters correlate with LVA presence or extent. Given the fact that many electrophysiologists incorporate voltage mapping to guide AF ablation to improve results, and presuming that patients without evidence of LVA may be sufficiently treated with PVI alone, this study provides important new insight into the promise: 1) Patients with LSPAF should not be excluded from voltage map-guided ablation procedures on the basis of long AF duration, advanced age, LA enlargement or fine AF ECG pattern; 2) Many LSPAF patients do not require voltage-derived substrate modification following PVI and therefore can avoid excessive ablation; 3) Simple non-invasive screening of the LSPAF population could predict LVA prevalence and help in further decision making.

However, it is still unclear if voltage-defined fibrosis presence and its extent can be useful markers in a decision as to whether a patient requires additional PV ablation.

\section{Funding}

The project was financed from the program of the Minister of Science and Higher Education under the name "Regional Initiative of Excellence" in 2019-2022 project number 002/RID/2018/19 amount of financing 12,000,000 PLN.

Conflict of interest: Radoslaw M. Kiedrowicz has received fellowship and educational support, travel grants and compensation for proctoring services from Biosence-Webster. Maciej Wielusinski has received educational support, travel grants and compensation for proctoring services from Biosence-Webster. Jaroslaw Kazmierczak has received travel grants and educational support from Biosence-Webster. Andrzej Wojtarowicz declares no conflict of interest.

\section{References}

1. Blandino A, Bianchi F, Grossi S, et al. Left atrial substrate modification targeting low-voltage areas for catheter ablation of atrial fibrillation: a systematic review and meta-analysis. Pacing Clin Electrophysiol. 2017; 40(2): 199-212, doi: 10.1111/pace.13015, indexed in Pubmed: 28054377.

2. Lang RM, Badano LP, Mor-Avi V, et al. Recommendations for cardiac chamber quantification by echocardiography in adults: an update from the American Society of Echocardiography and the European Association of Cardiovascular Imaging. Eur Heart J Cardiovasc Imaging. 2015; 16(3): 233-270, doi: 10.1093/ehjci/ jev014, indexed in Pubmed: 25712077.

3. Nault I, Lellouche N, Matsuo S, et al. Clinical value of fibrillatory wave amplitude on surface ECG in patients with persistent atrial fibrillation. J Interv Card Electrophysiol. 2009; 26(1): 11-19, doi: 10.1007/s10840-009-9398-3, indexed in Pubmed: 19404588.

4. Rolf S, Kircher S, Arya A, et al. Tailored atrial substrate modification based on low-voltage areas in catheter ablation of atrial fibrillation. Circ Arrhythm Electrophysiol. 2014; 7(5): 825-833, doi: 10.1161/CIRCEP.113.001251, indexed in Pubmed: 25151631.

5. Huo Y, Gaspar T, Pohl M, et al. Prevalence and predictors of low voltage zones in the left atrium in patients with atrial fibrillation. Europace. 2018; 20(6): 956-962, doi: 10.1093/europace/eux082, indexed in Pubmed: 28605524.

6. Vlachos K, Efremidis M, Letsas KP, et al. Low-voltage areas detected by high-density electroanatomical mapping predict recurrence after ablation for paroxysmal atrial fibrillation. J Cardiovasc Electrophysiol. 2017; 28(12): 1393-1402, doi: 10.1111/jce.13321, indexed in Pubmed: 28884923.

7. Masuda M, Fujita M, Iida O, et al. Left atrial low-voltage areas predict atrial fibrillation recurrence after catheter ablation in patients with paroxysmal atrial fibrillation. Int J Cardiol. 2018; 257: 97-101, doi: 10.1016/j.ijcard.2017.12.089, indexed in Pubmed: 29506746.

8. Wang XH, Li Z, Mao JL, et al. Low voltage areas in paroxysmal atrial fibrillation: The prevalence, risk factors and impact on the effectiveness of catheter ablation. Int J Cardiol. 2018; 269: 139-144, doi: 10.1016/j.ijcard.2018.07.076, indexed in Pubmed: 30060968.

9. Zhou W, Wang L, Zhou Bo, et al. Catheter ablation of paroxysmal atrial fibrillation using high-density mapping-guided substrate modification. Pacing Clin Electrophysiol. 2018; 41(12): 1630-1634, doi: 10.1111/pace.13524, indexed in Pubmed: 30353561. 
10. Wang XH, Li Z, Mao JL, et al. A novel individualized substrate modification approach for the treatment of long-standing persistent atrial fibrillation: preliminary results. Int J Cardiol. 2014; 175(1): 162-168, doi: 10.1016/j.ijcard.2014.05.022, indexed in Pubmed: 24874911.

11. Yang G, Yang B, Wei Y, et al. Catheter ablation of nonparoxysmal atrial fibrillation using electrophysiologically guided substrate modification during sinus rhythm after pulmonary vein isolation. Circ Arrhythm Electrophysiol. 2016; 9(2): e003382, doi: 10.1161/ CIRCEP.115.003382, indexed in Pubmed: 26857907.

12. Jadidi AS, Lehrmann H, Keyl C, et al. Ablation of persistent atrial fibrillation targeting low-voltage areas with selective activation characteristics. Circ Arrhythm Electrophysiol. 2016; 9(3), doi: 10.1161/CIRCEP.115.002962, indexed in Pubmed: 26966286.

13. Yamaguchi T, Tsuchiya T, Fukui A, et al. Efficacy of left atrial voltage-based catheter ablation of persistent atrial fibrillation. J Cardiovasc Electrophysiol. 2016; 27(9): 1055-1063, doi: 10.1111/jce.13019, indexed in Pubmed: 27235000.

14. Yang B, Jiang C, Lin Y, et al. STABLE-SR (Electrophysiological Substrate Ablation in the Left Atrium During Sinus Rhythm) for the Treatment of Nonparoxysmal Atrial Fibrillation: A Prospective, Multicenter Randomized Clinical Trial. Circ Arrhythm Electrophysiol. 2017; 10(11), doi: 10.1161/CIRCEP.117.005405, indexed in Pubmed: 29141843.

15. Ammar-Busch S, Buiatti A, Tatzber A, et al. Predictors of low voltage areas in persistent atrial fibrillation: is it really a matter of time? J Interv Card Electrophysiol. 2020; 57(3): 345-352, doi: 10.1007/s10840-018-0471-7, indexed in Pubmed: 30374659.

16. Kumagai K, Toyama H, Zhang Bo. Effects of additional ablation of low-voltage areas after Box isolation for persistent atrial fibrillation. J Arrhythm. 2019; 35(2): 197-204, doi: 10.1002/joa3.12169, indexed in Pubmed: 31007783.

17. Kottkamp H, Berg J, Bender R, et al. Box isolation of fibrotic areas (BIFA): a patient-tailored substrate modification approach for ablation of atrial fibrillation. J Cardiovasc Electrophysiol. 2016; 27(1): 22-30, doi: 10.1111/jce.12870, indexed in Pubmed: 26511713.

18. Yagishita A, Gimbel JR, De Oliveira S, et al. Long-Term outcome of left atrial voltage-guided substrate ablation during atrial fibrillation: a novel adjunctive ablation strategy. J Cardiovasc Electrophysiol. 2017; 28(2): 147-155, doi: 10.1111/jce.13122, indexed in Pubmed: 27862561.

19. Kircher S, Arya A, Altmann D, et al. Individually tailored vs. standardized substrate modification during radiofrequency catheter ablation for atrial fibrillation: a randomized study. Europace. 2018; 20(11): 1766-1775, doi: 10.1093/europace/eux310, indexed in Pubmed: 29177475.

20. Schreiber D, Rieger A, Moser F, et al. Catheter ablation of atrial fibrillation with box isolation of fibrotic areas: Lessons on fibrosis distribution and extent, clinical characteristics, and their impact on long-term outcome. J Cardiovasc Electrophysiol. 2017; 28(9): 971-983, doi: 10.1111/jce.13278, indexed in Pubmed: 28635186.

21. Huang D, Li JB, Zghaib T, et al. The Extent of Left Atrial LowVoltage Areas Included in Pulmonary Vein Isolation Is Associated With Freedom from Recurrent Atrial Arrhythmia. Can J Cardiol. 2018; 34(1): 73-79, doi: 10.1016/j.cjca.2017.10.012, indexed in Pubmed: 29275886.

22. Ahmed-Jushuf F, Murgatroyd F, Dhillon P, et al. The impact of the presence of left atrial low voltage areas on outcomes from pulmonary vein isolation. J Arrhythm. 2019; 35(2): 205-214, doi: 10.1002/joa3.12174, indexed in Pubmed: 31007784.
23. Matsuda Y, Masuda M, Asai M, et al. Impact of renal dysfunction on left atrial low-voltage areas in patients with atrial fibrillation. Circ J. 2019; 83(5): 985-990, doi: 10.1253/circj.CJ-18-1277, indexed in Pubmed: 30853681.

24. Kosiuk J, Dinov B, Kornej J, et al. Prospective, multicenter validation of a clinical risk score for left atrial arrhythmogenic substrate based on voltage analysis: DR-FLASH score. Heart Rhythm. 2015; 12(11): 2207-2212, doi: 10.1016/j. hrthm.2015.07.003, indexed in Pubmed: 26144350.

25. Nery PB, Al Dawood W, Nair GM, et al. Characterization of lowvoltage areas in patients with atrial fibrillation: insights from high-density intracardiac mapping. Can J Cardiol. 2018; 34(8): 1033-1040, doi: 10.1016/j.cjca.2018.04.008, indexed in Pubmed: 30056843.

26. Kishima H, Mine T, Fukuhara E, et al. The association between left atrial stiffness and low-voltage areas of left atrium in patients with atrial fibrillation. Heart Vessels. 2019; 34(11): 1830-1838, doi: 10.1007/s00380-019-01423-z, indexed in Pubmed: 31049675.

27. Hohendanner F, Romero I, Blaschke F, et al. Extent and magnitude of low-voltage areas assessed by ultra-high-density electroanatomical mapping correlate with left atrial function. Int J Cardiol. 2018; 272: 108-112, doi: 10.1016/j.ijcard.2018.07.048, indexed in Pubmed: 30017527.

28. Wijffels MC, Kirchhof CJ, Dorland R, et al. Atrial fibrillation begets atrial fibrillation. A study in awake chronically instrumented goats. Circulation. 1995; 92(7): 1954-1968, doi: 10.1161/01. cir.92.7.1954, indexed in Pubmed: 7671380.

29. Teh AW, Kistler PM, Lee G, et al. Long-term effects of catheter ablation for lone atrial fibrillation: progressive atrial electroanatomic substrate remodeling despite successful ablation. Heart Rhythm. 2012; 9(4): 473-480, doi: 10.1016/j.hrthm.2011.11.013, indexed in Pubmed: 22079885.

30. Silbiger JJ. Mechanistic insights into atrial functional mitral regurgitation: Far more complicated than just left atrial remodeling. Echocardiography. 2019; 36(1): 164-169, doi: 10.1111/ echo.14249, indexed in Pubmed: 30620100.

31. Iacuzio L, Essayagh B, Civaia F, et al. Right-Sided heart structural and functional remodeling in mitral regurgitation secondary to mitral valve prolapse. Am J Cardiol. 2018; 122(12): 2095-2103, doi: 10.1016/j.amjcard.2018.08.062, indexed in Pubmed: 30293652.

32. Begg GA, Lip GYH, Plein S, et al. Circulating biomarkers of fibrosis and cardioversion of atrial fibrillation: A prospective, controlled cohort study. Clin Biochem. 2017; 50(1-2): 11-15, doi: 10.1016/j.clinbiochem.2016.09.008, indexed in Pubmed: 27622867.

33. Patel D, Mohanty P, Di Biase L, et al. Outcomes and complications of catheter ablation for atrial fibrillation in females. Heart Rhythm. 2010; 7(2): 167-172, doi: 10.1016/j.hrthm.2009.10.025, indexed in Pubmed: 20022814.

34. Park JH, Joung B, Son NH, et al. The electroanatomical remodelling of the left atrium is related to CHADS2/CHA2DS2VASc score and events of stroke in patients with atrial fibrillation. Europace. 2011; 13(11): 1541-1549, doi: 10.1093/europace/eur135, indexed in Pubmed: 21576128.

35. Chen J, Arentz T, Cochet H, et al. Extent and spatial distribution of left atrial arrhythmogenic sites, late gadolinium enhancement at magnetic resonance imaging, and low-voltage areas in patients with persistent atrial fibrillation: comparison of imaging vs. electrical parameters of fibrosis and arrhythmogenesis. Europace. 2019; 21(10): 1484-1493, doi: 10.1093/europace/euz159, indexed in Pubmed: 31280323. 